\title{
Nonlocal Dynamics of Spontaneous Imbibition Fronts
}

\author{
Dimitrios Geromichalos, ${ }^{*}$ Frieder Mugele, and Stephan Herminghaus \\ Applied Physics Lab, Ulm University, Ulm, D-89069, Germany
}

(Received 1 March 2002; published 19 August 2002)

\begin{abstract}
We have studied spontaneous imbibition fronts generated by capillary rise between two roughened glass plates, the separation $d$ of which varied between 10 and $50 \mu \mathrm{m}$. Perfect agreement with Washburn's law was obtained. We have determined the roughness exponent $\chi$ of the fronts, and found $\chi=0.81 \pm 0.01$ for small length scales. Above a certain crossover length $\xi$, it reached $\chi=0.58 \pm 0.04$, as predicted by the quenched noise Kardar-Parisi-Zhang equation. The crossover length is found to scale with the plate separation as $\sqrt{d}$, as predicted by recent models which properly include nonlocal dynamics effects on the front. We believe this to be the first clear identification of crossover from nonlocal to local dynamics.
\end{abstract}

The process of imbibition of a liquid in a random medium plays a key role in many problems of general interest, such as oil recovery, irrigation in agriculture, or wetting of raw material powders in industrial chemistry. Inspired by this tremendous practical importance, many experiments have already been carried out in search of a thorough physical understanding of the basic mechanisms involved. However, a satisfactory agreement with theory has not yet been achieved. This is in part due to difficulties inherent to the experiments performed so far. Well-known problems of imbibition experiments which use paper as the random medium [1-3] are swelling, poorly defined roughness, and evaporation [4]. Other experiments using HeleShaw cells with randomly distributed glass beads $[5,6]$ or columns with glass beads [7] provide a well-defined geometry, but exhibit strong pinning effects and rather large intrinsic length scales (as the diameter of the beads), such that important statistical features of the invading front may escape observation.

Theoretical approaches were mainly concerned with scaling arguments, which accounted for nonlocal aspects of the flow dynamics at best qualitatively. In recent years, however, there has been considerable improvement in the theoretical description of advancing liquid front phenomena [8-11], with particular focus on the nonlocal effects on imbibition fronts. These come about from lateral pressure gradients close to the front line, and strongly affect the statistics of the dynamical front roughness. It thus seems worthwhile to revisit the imbibition problem from the experimental side.

We have studied spontaneous imbibition of a liquid front into the gap between two roughened glass plates. This geometry bears some reminiscence to some of the earlier Hele-Shaw cell experiments, but is designed in particular, to reduce pinning effects, for reasons which will become clear below. The plates were made from optical quality glass, purchased with a specified planity of about $1 \mu \mathrm{m}$ over a distance of $10 \mathrm{~cm}$. The thickness of the plates was chosen to be as thick as $8 \mathrm{~mm}$, in order to avoid any bending of the glass surfaces by capillary forces. Of each plate, one surface was roughened by lapping with coarse sapphire powder, thereby conserving the large scale planity. The maximum height variation in the topography of the rough surfaces was $\approx 3 \mu \mathrm{m}$. This can be seen in Fig. 1(a), which was obtained by scanning force microscopy (SFM). The Fourier transform of the topography (Fig. 1(b)) shows the homogeneity and isotropy of the roughness. In order to demonstrate this on a larger scale, we illuminated the rough glass plate with an expanded laser beam, and focused the transmitted light onto a CCD camera chip [12]. The smooth intensity distribution, which is shown in
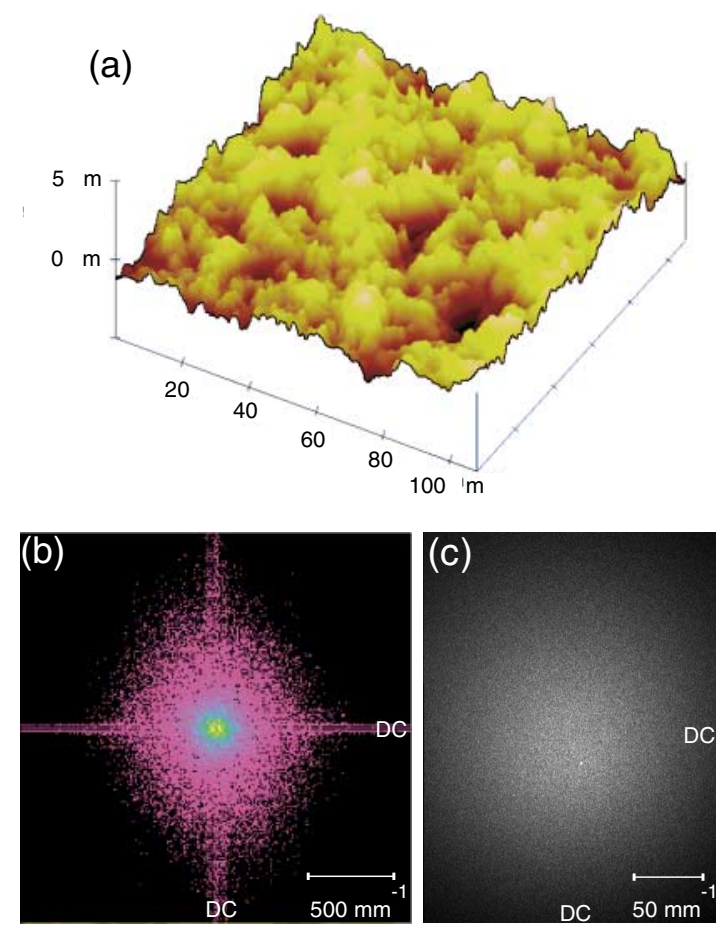

FIG. 1 (color online). (a) SFM topography of the roughened surface of one of the glass plates. (b) Fourier transform of the SFM picture. The cross lines are artifacts due to the transform procedure. (c) Optical Fourier transform, showing the roughness on larger length scales. 
Fig. 1(c), demonstrates the large scale isotropy of the roughness.

Before the experiment, the glass plates were ultrasonicated subsequently with acetone, ethanol, and water, and blown dry with nitrogen gas. In order to remove possible residual organic contamination, they were immersed for several hours in concentrated sulfuric acid with an oxidant added, thoroughly washed with deionized water, and blown dry with nitrogen again.

The plates were mounted with the rough sides facing each other, using stainless steel ribbons with thickness ranging from 10 to $50 \mu \mathrm{m}$ as spacers, in order to maintain a well-defined plate separation. Spontaneous imbibition was initiated by placing the lower edge of the vertically mounted pair of plates into a liquid reservoir. The liquid invaded the gap between the plates spontaneously due to capillary forces. The plates were illuminated with a white light source and observed in reflection with a high resolution CCD camera. Optical immersion of the roughness by the fluid yielded a sharp contrast at the invading front line. Five runs were taken at each plate separation to improve the statistics. Depending on the plate separation, the boundary line was disturbed within a few $\mu \mathrm{m}$ from the edges of the plates (total width $\approx 7 \mathrm{~cm}$ ). In order to avoid these edge effects, we analyzed only the central part (width $\approx 3 \mathrm{~cm}$ ) of the moving fronts.

Water and 1-undecanol $\left[\mathrm{CH}_{3}\left(\mathrm{CH}_{2}\right)_{10} \mathrm{OH}\right]$ were used as the wetting liquids. The latter yields particularly smooth fronts which may easily be analyzed by conventional procedures. In the case of water, air entrainment at the moving front, leading to the inclusion of air bubbles in the rising liquid column, is more likely to occur. This phenomenon is interesting for itself, but will not be discussed in the present Letter. Furthermore, the comparably high viscosity of undecanol gives rise to a smaller front velocity, thereby facilitating data acquisition.

A typical front obtained with undecanol is displayed in Fig. 2(a). It was generally observed that for larger plate separation $d$, the fronts became smoother: This can be attributed to the increased influence of the front line tension, which is directly proportional to the plate separation. For all plate separations investigated, the height of the front was small as compared to the total capillary ascension. In this case, we would expect the average height of the front to rise according to Washburn's law, i.e., $H=A \sqrt{t}$ [13], if all parameters are uniform throughout the slit. In fact, this is fulfilled with satisfactory precision, as it is demonstrated ion Fig. 2(b). The prefactors $A$ found experimentally were constantly about one-half the corresponding theoretical values for smooth plates and perfect wetting, $A_{t h}=$ $\sqrt{d \gamma / 3 \eta}$. The reason for this discrepancy is very likely the modification of the hydrodynamic boundary conditions at the glass due to the roughness, and possible small scale local variations in surface energy of the roughened glass.

The invading front line was imaged using a high resolution CCD camera, digitized and subsequently analyzed
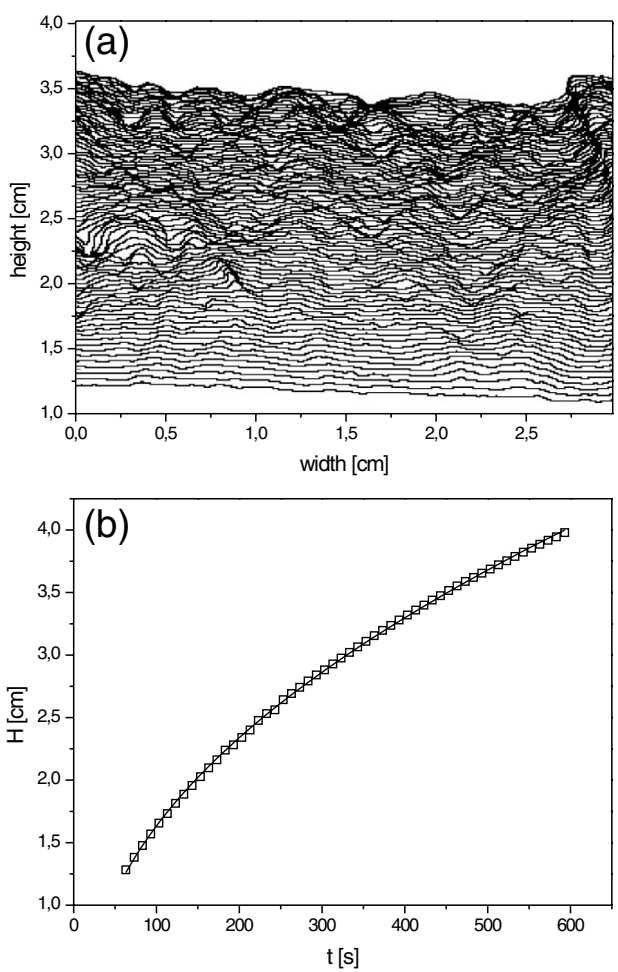

FIG. 2. Rising undecanol front, with a glass plate separation of $10 \mu \mathrm{m}$ : (a) The front at a series of equidistant times. The time elapsed between two snapshots is $10 \mathrm{sec}$. (b) The median height $H$ of the front as a function of time. Washburn's law is superimposed as a solid line, in good agreement with the data.

numerically. The roughness exponent $\chi[14]$ was obtained from the correlation function

$$
C(l)=\sqrt{\left\langle|h(x+l)-h(x)|^{2}\right\rangle},
$$

where $h(x)$ is the vertical position of the front, and $x$ is the lateral coordinate. The mediation went over the whole contact line and was carried out for several measurements. The scale dependent roughness exponent of the front was defined via $\chi:=\frac{d \ln C(l)}{d \ln l}$.

In order to obtain results which are comparable and suited to check the validity of the phase field model (see below), we limited our analysis to fronts with a mean height $H$ from 2.5 to $3.5 \mathrm{~cm}$. Some typical results are sketched in Fig. 3(a). As is clearly visible, two different roughness exponents for different length scales exist. For distances smaller than a certain length $\xi$, we obtain $\chi_{1} \approx$ 0.8 which crosses over to the quenched noise KardarParisi-Zhang $(\mathrm{QKPZ})$ exponent $\chi_{2} \approx 0.6[15,16]$ for larger distances. The latter regime is indicative of a local dynamics, i.e., a dynamics affected by lateral interactions only over distances small as compared to the probed length scale. In this case, the occurrence of the QKPZ exponent is rather ubiquitous, and may be viewed as a test of the accuracy of the experiments. 

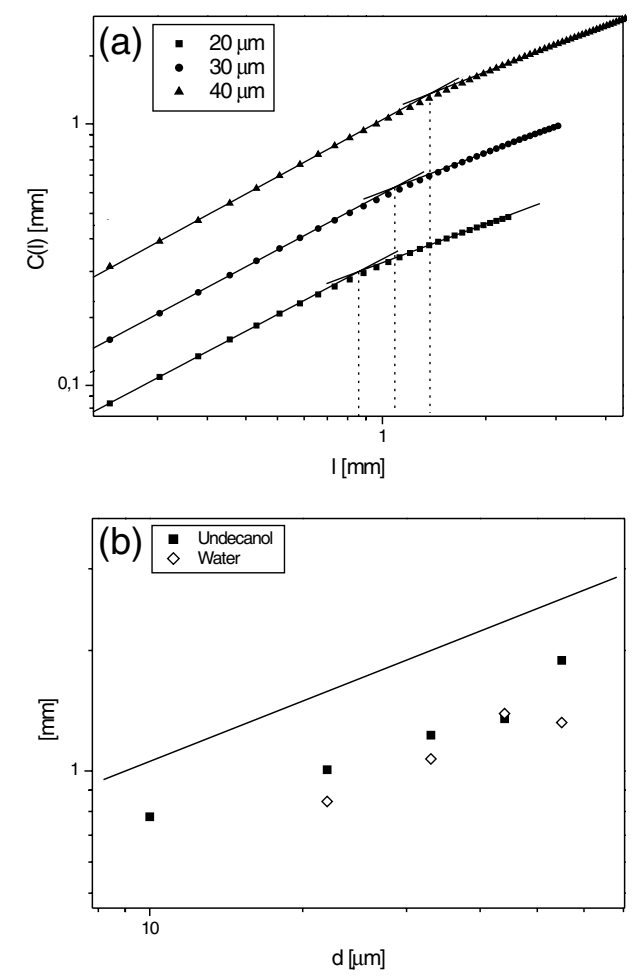

FIG. 3. (a) Examples for spatial correlation functions with two regimes for water and different plate separations (20 to $40 \mu \mathrm{m})$ (The curves for 30 and $40 \mu \mathrm{m}$ have been shifted for clarity.) The values of $\chi$ in these cases cross over with rising plate separations from 0.82 to 0.60 , from 0.81 to 0.60 , and from 0.80 to 0.57 , respectively. The dotted lines mark the values of the crossover lengths. (b) Crossover lengths $\xi$ for different plate separations. Closed symbols: undecanol. Open symbols: water. The solid curve indicates the theoretical prediction, $\xi \propto \sqrt{d}$.

The regime observed at smaller scales is particularly interesting, since it deviates from the QKPZ behavior. While the locality of the dynamics can in principle be obeyed on all length scales in a system such as, for instance, moving magnetic domain boundaries, it is clear the conservation of mass must be taken into account in the case of a moving liquid front. The latter unavoidably leads to nonlocal effects on short length scales because the liquid required to advance the front at one place must be taken from the neighborhood. However, communication between adjacent locations will be limited to some characteristic length $\xi$, which determines the transition from short range nonlocal to long range local (i.e., KPZ) behavior. Recently, theories have been developed which appropriately incorporate nonlocal effects due to lateral pressure gradients in the liquid close to the front line. These are accounted for by Darcy's law, $\mathbf{v}=-\kappa \nabla p$, which describes two-phase flows in porous media and connects the pressure gradient $\nabla p$ with the velocity field $\mathbf{v}$. An equation of motion of the fluid front may be derived [8] invoking fluid incompressibility, $\nabla \mathbf{v}=0$, and using the Grinstein-Ma expression of the random field Ising model,

$$
\frac{\partial h}{\partial t}=F+\gamma \frac{\partial^{2} h}{\partial x^{2}}+\eta(x, h),
$$

wherein $F$ is the driving force, $h$ the height of the front, $x$ the horizontal position, $\gamma$ the surface tension, and $\eta$ a term describing the roughness ("quenched noise"; [8] and references therein). The equation of motion, as obtained by Ganesan and Brenner [8], is intrinsically nonlocal, nonlinear, and rather complicated, but scaling properties of its solutions may be derived for small amplitudes $h(x, t)-$ $H(t)$. It was found (for forced fluid invasion, FFI) that $\chi_{1}=$ 0.75 , which is remarkably close to our result. For spontaneous imbibition, the authors give a roughness exponent of 0.633 which is the expected value for strong pinning by directed percolation [16]. However, the experiments reported here fall in the FFI universality class, since there was practically no pinning observed.

Indications of similar crossover effects have been reported before, but were interpreted in terms of pinning effects [1]. The main difference to our experiment is that the roughness amlitude was rather large, since the front advanced through a layer of glass beads. In this case, pinning may indeed be stronger, and mask the intrinsic nonlocal effects. It may nevertheless be worthwhile to reexamine these results in view of the more advanced theories which are available now. In our experiments, pinning is of minor importance, such that the large roughness exponent we observed at small scales is a good candidate for an intrinsic nonlocality fingerprint. In order to establish this, however, we must seek further evidence.

The phase field model $[10,17]$ casts the problem of fluid invasion into a Ginzburg-Landau-like scheme, where the dry and the wetted state are considered as two "phases" of the system. This model is elaborate enough to derive more information about the crossover length $\xi$, and its dependence on experimental parameters. For spontaneous imbibition, it predicts that the crossover length $\xi$ should be time dependent [10], since $\xi=\sqrt{(\gamma / 6 \eta v)} d$ and $v \propto 1 / \sqrt{t}$. This dependence $\left(\propto t^{1 / 4}\right)$ is too weak to be detectable in our experiments. However, since for the mean velocity $v$ we have $v \propto \sqrt{(d \gamma / 3 \eta)}(1 / \sqrt{t})$, due to Washburn's law, and since we analyzed only fronts within a small and fixed range of $H$, the crossover length becomes

$$
\xi \propto \sqrt{d} .
$$

Indeed, as it can be seen in Fig. 3(b), $\xi$ clearly rises with the plate distance. The solid line indicates the expected slope of 0.6. On the basis of the phase field model, we can roughly estimate $\xi$ from the measured front velocity to be of the order of $2 \mathrm{~mm}$. This corresponds quite well to the values found in our analysis, as displayed in Fig. 3(b). We may assume, on the basis of this evidence, that we have observed both the nonlocal and the local regime of liquid invasion into a disordered medium in $2 \mathrm{D}$, and the crossover between both. 


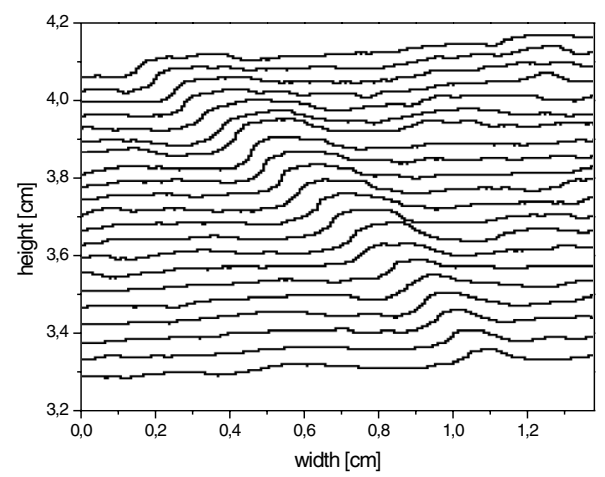

FIG. 4. Rising undecanol front, with a glass plate separation of $10 \mu \mathrm{m}$ at a series of equidistant times. $(\Delta t=4 \mathrm{sec})$

As mentioned above, the equation of motion derived in Ref. [8] is strongly nonlinear, and the nature of its solutions at larger amplitude has not yet been investigated. It is therefore interesting to note that we have frequently observed kinks in the advancing front to move laterally, such as one would expect from a solitonic solution to the equation of motion. Figure 4 shows a series of front lines superimposed as in Fig. 2(a), displaying such an object. It is seen to move from right to left, with a velocity which is comparable to the velocity of the front. We were not yet able to initiate these effects in a controlled way, and found the lateral propagation velocities to scatter significantly. Nevertheless, it may be of interest for theorists to search for solitonic solutions in the equation of motion of the front, such as the one proposed in Ref. [8].

In conclusion, we have observed the crossover from nonlocal to QKPZ behavior in liquid imbibition. We determined the roughness exponent $\chi$ of the fronts and found that it crossed over from a value of 0.8 at small scales to the value of 0.6 . We were also able to vary the crossover length by changing experimental parameters. This demonstrates for the first time the validity of recent theoretical treatments proposed for liquid imbibition in a disordered medium.

The authors appreciate inspiring discussions with Tapio Ala-Nissila and Sami Majaniemi from Helsinki University.
Financial support from the German Science Foundation within the Priority Program "Wetting and Structure Formation at Interfaces" is gratefully acknowledged.

*Electronic address: Dimitrios.Geromichalos@physik.uniulm.de

[1] V. K. Horváth, F. Family, and T. Vicsek, Phys. Rev. Lett. 67, 3207 (1991).

[2] S. V. Buldyrev, A.-L. Barabási, F. Caserta, S. Havlin, H. E. Stanley, and T. Vicsek, Phys. Rev. A 45, R8313 (1992).

[3] V. K. Horváth and H. E. Stanley, Phys. Rev. E 52, 5166 (1995).

[4] M. Dubé, M. Rost, and M. Alava, Eur. Phys. J. B 15, 691 (2000).

[5] V. K. Horváth, F. Family, and T. Vicsek, J. Phys. A 24, L25 (1991).

[6] A. Paterson, M. Fermigier, P. Jenffer, and L. Limat, Phys. Rev. E 51, 1291 (1995).

[7] T. Delker, D. B. Pengra, and P.-Z. Wong, Phys. Rev. Lett. 76, 2902 (1996).

[8] V. Ganesan and H. Brenner, Phys. Rev. Lett. 81, 578 (1998).

[9] C.-H. Lam and V. K. Horváth, Phys. Rev. Lett. 85, 1238 (2000).

[10] M. Dubé, M. Rost, K. R. Elder, M. Alava, S. Majaniemi, and T. Ala-Nissila, Phys. Rev. Lett. 83, 1628 (1999).

[11] A. Hernández-Machado, J. Soriano, A. M. Lacasta, M. A. Rodriguez, L. Ramirez-Piscina, and J. Ortin, Europhys. Lett. 55, 194 (2001).

[12] E. Hecht, Optics (Addison-Wesley, New York, 1998), 3rd ed.

[13] E. W. Washburn, Phys. Rev. 17, 273 (1921).

[14] F. Family and T. Vicsek, J. Phys. A 18, L75 (1985).

[15] M. Kardar, G. Parisi, and Y.-C. Zhang, Phys. Rev. Lett. 56, 889 (1986).

[16] A.-L. Barabási and H. E. Stanley, Fractal Concepts in Surface Growth (Cambridge University Press, Cambridge, United Kingdom, 1995).

[17] M. Dubé, M. Rost, K. R. Elder, M. Alava, S. Majaniemi, and T. Ala-Nissila, Eur. Phys. J. B 15, 701 (2000). 